\title{
DENSITY RELATIVE TO A TORSION THEORY
}

\author{
PAUL BLAND AND STEPHEN RILEY
}

\begin{abstract}
If $(\mathcal{T}, \mathcal{F})$ is a torsion theory on $\operatorname{Mod} R$, then a ring $B$ of biendomorphisms of a $\mathfrak{T}$-cocritical module is topologized. Moreover, if a certain factor module of $R$ is quasi-projective, a ring monomorphism $\varphi: R \rightarrow B$ is found such that $\varphi(R)$ is topologically dense in $B$. This is done in such a way that when $(\mathscr{T}, \mathscr{F})$ is the torsion theory in which every module is torsion free, the Jacobson density theorem is recovered.
\end{abstract}

Throughout this paper, $R$ will denote an associative ring with identity and $\operatorname{Mod} R$ will stand for the category of unital right $R$-modules. Unless stated otherwise, all mappings will be morphisms in a module category and morphisms will be written on the side of the argument opposite that of scalars.

In [1], Dickson defined a torsion theory on $\operatorname{Mod} R$ to be a pair $(\mathcal{T}, \mathcal{F})$ of classes of right $\boldsymbol{R}$-modules such that:

(a) $\mathcal{T} \cap \mathcal{F}=0$.

(b) If $M \rightarrow N \rightarrow 0$ is exact and $M \in \mathcal{T}$, then $N \in \mathcal{T}$.

(c) If $0 \rightarrow M \rightarrow N$ is exact and $N \in \mathcal{F}$, then $M \in \mathscr{F}$.

(d) For each module $M$, there is an exact sequence $0 \rightarrow L \rightarrow M \rightarrow N \rightarrow 0$ where $L \in \mathcal{T}$ and $N \in \mathcal{F}$. Modules in $\mathcal{T}$ are called torsion and those in $\mathcal{F}$ torsion free.

If $(\mathcal{T}, \mathcal{F})$ is a torsion theory on Mod $R$, then $\mathcal{T}$ is closed under isomorphic images, factors, extensions, and direct sums while $\mathscr{F}$ is closed under isomorphic images, submodules, extensions, and direct products [1, Theorem 2.3]. By saying that a class $\mathcal{C}$ of modules is closed under extensions we mean that if $N$ is a submodule of $M$ such that $N$ and $M / N$ are in $\mathcal{C}$, then $M$ is in $\mathcal{C}$. ( $\mathcal{T}, \mathcal{F}$ ) will now denote a fixed but arbitrary torsion theory on $\operatorname{Mod} R$. The interested reader can consult [2], [5] or [7] for a more extensive development of torsion theories.

A submodule $N$ of $M$ will be called pure in $M$ if $M / N$ is torsion free and following Golan [2, p. 181] we call a nonzero module $M \mathcal{T}$-cocritical if $M$ is torsion free and every proper homomorphic image of $M$ is torsion. A right ideal $K$ of $R$ is called $\mathscr{T}$-critical if $R / K$ is a $\mathscr{T}$-cocritical right $R$-module. It is not difficult to show that $K$ is a $\mathscr{T}$-critical right ideal of $R$ if and only if $K$ is maximal among the pure right ideals of $R$. Thus, when $(\mathcal{T}, \mathcal{F})$ is the torsion theory in which every module is torsion free, the $\mathcal{T}$-critical right ideals of $R$ are just the maximal right ideals of $R$ and the $\mathcal{T}$-cocritical modules are just the simple right $R$-modules. Hence, the

Received by the editors January 29, 1980.

AMS (MOS) subject classifications (1970). Primary 16A63, 16A20; Secondary 18E40.

Key words and phrases. Torsion theory, $\mathcal{T}$-cocritical module, $\mathcal{T}$-critical right ideal, right $\mathcal{T}$-primitive ring, density theorem. 
$\mathcal{T}$-critical right ideals of $R$ and the $\mathcal{T}$-cocritical right $R$-modules are in some sense "generalized maximal" right ideals of $R$ and "generalized simple" right $R$-modules respectively. Since the endomorphism ring of a $\mathcal{T}$-cocritical module $M$ is a (noncommutative) integral domain [2, Proposition 18.2], $M$ is, in the sense described above, a "generalized left vector space" over the endomorphism ring of $M$. Consequently, one is led to consider the questions: Can one topologize a ring $B$ of biendomorphisms of a $\mathcal{T}$-cocritical module $M$ and find a ring monomorphism $\varphi$ : $R \rightarrow B$ such that $\varphi(R)$ is topologically dense in $B$ ? Moreover, can this be done in such a fashion that when $(\mathcal{F}, \mathcal{F})$ is the torsion theory in which every module is torsion free, the Jacobson density theorem is recovered? The purpose of this paper is to answer these questions in the affirmative subject to a certain factor module of $R$ being quasi-projective as a right $R$-module.

An ideal $I$ of $R$ is right $\mathcal{T}$-primitive if it is the largest ideal contained in some $\mathcal{T}$-critical right ideal of $R$, while $R$ will be called right $\mathcal{T}$-primitive if (0) is a right $\mathcal{T}$-primitive ideal of $R$. It now follows that $I$ is a right $\mathcal{T}$-primitive ideal of $R$ if and only if $I=(K: R)^{1}$ for some $\mathcal{T}$-critical right ideal $K$ of $R$. An easy adaptation of the proof of $[6$, p. 52, Proposition 2] yields the following

PROPOSITION 1. $R$ is right $\mathcal{T}$-primitive if and only if $R$ admits a faithful, cyclic, T-cocritical right $R$-module.

It is now immediate that if $R$ is right $\mathscr{T}$-primitive, then $R$ is torsion free. This

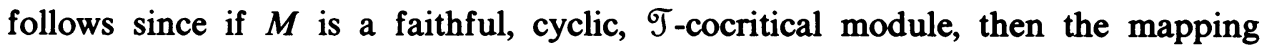
$R \rightarrow \Pi_{x \in M} M_{x}: r \rightarrow(x r)$ is an $R$-linear embedding where $M_{x}=M$ for each $x \in$ $M$.

Now suppose that $R$ is a right $\mathcal{T}$-primitive ring and that (0) is the largest ideal

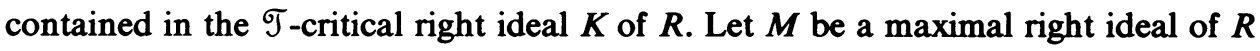
which contains $K$ and set $R^{*}=R / K, E=\operatorname{End}\left(R_{R}^{*}\right), M^{*}=M / K$ and $B=\{g \in$ $\left.\operatorname{End}\left({ }_{E} R^{*}\right) \mid M^{*} g \subseteq M^{*}\right\}$. Since $M^{*} r \subseteq M^{*}$ for each $r \in R$, the canonical ring homomorphism $\varphi: R \rightarrow B: r \rightarrow \tau_{r}$ where $\tau_{r}: R^{*} \rightarrow R^{*}: x \rightarrow x r$ does indeed have codomain $B$ and it is an embedding since $R^{*}$ is faithful. We now assume that $R$ is right $\mathcal{T}$-primitive and that $K, M^{*}, R^{*}$, and $B$ are as above.

In order to topologize $B$, for any $f \in B$ and $x \in R^{*}$, let $O(x, x f)$ be the set $\left\{g \in B \mid x g-x f \in M^{*}\right\}$. As $x$ varies throughout $R^{*}$, the collection of all such $O(x, x f)$ forms a subbase for a neighborhood system of $f$. We call the resulting topology the $M^{*}$-topology on $B$. Notice that since $(M: R) \simeq \cap_{x \in R^{*}} O(x, 0), R$ is right primitive if and only if the $M^{*}$-topology on $B$ is Hausdorff. Using an argument similar to that in the proof of $[3$, p. 29, Proposition 3] one can prove

Proposition 2. B is a topological ring when endowed with the $M^{*}$-topology.

A set $\left\{x_{1}, x_{2}, \ldots, x_{n}\right\}_{n}$ of distinct elements of $R^{*}$ is said to be $M^{*}$-independent if $x_{j} \cap_{i=1 ; i \neq j}\left(M^{*}: x_{i}\right) \nsubseteq M^{*}$ for $j=1,2, \ldots, n$.

\footnotetext{
${ }^{1}$ If $A$ and $B$ are nonempty subsets of a module $M$, then $(A: B)=\{r \in R \mid B r \subseteq A\}$. If $B=\{x\}$, we write $(A: x)$ for $(A:\{x\})$.
} 
LEMMA 3. The set $\left\{x_{1}, x_{2}, \ldots, x_{n}\right\}$ of elements of $R^{*}$ is $M^{*}$-independent if and only if $\left\{x_{1}+M^{*}, x_{2}+M^{*}, \ldots, x_{n}+M^{*}\right\}$ is a linearly independent set of vectors in the left $\operatorname{End}\left(R^{*} / M^{*}\right)$-vector space $R^{*} / M^{*}$.

Proof. Let $\Delta=\operatorname{End}\left(R^{*} / M^{*}\right)$ and if $S$ is a nonempty subset of $R^{*} / M^{*}$, set $S^{r}=\{r \in R \mid S r=0\}$ and $S^{r l}=\left\{x \in R^{*} / M^{*} \mid x S^{r}=0\right\}$. Now suppose that the set $\left\{x_{1}, x_{2}, \ldots, x_{n}\right\}$ is $M^{*}$-independent and that the vectors $x_{1}+M^{*}, x_{2}+$ $M^{*}, \ldots, x_{n}+M^{*}$ are $\Delta$-linearly dependent. Let $\sum_{i=1}^{n} k_{i}\left(x_{i}+M^{*}\right)=0$ in $R^{*} / M^{*}$ and suppose that $k_{j} \neq 0$; then $0 \neq k_{j}\left(x_{j}+M^{*}\right) \in \sum_{i=1 ; i \neq j}^{n} \Delta\left(x_{i}+M^{*}\right)$. Hence, it follows that

$$
\begin{aligned}
\left(x_{j}+M^{*}\right)^{r} & =\left[k_{j}\left(x_{j}+M^{*}\right)\right]^{r} \supseteq\left[\sum_{\substack{i=1 \\
i \neq j}}^{n} \Delta\left(x_{i}+M^{*}\right)\right]^{r} \\
& =\bigcap_{\substack{i=1 \\
i \neq j}}^{n}\left[\Delta\left(x_{i}+M^{*}\right)\right]^{r}=\bigcap_{\substack{i=1 \\
i \neq j}}^{n}\left(x_{i}+M^{*}\right)^{r} .
\end{aligned}
$$

Thus, $\left(x_{j}+M^{*}\right) \cap_{i=1 ; i \neq j}^{n}\left(x_{i}+M^{*}\right)^{r}=0$ in $R^{*} / M^{*}$ and so

$$
x_{j} \bigcap_{\substack{i=1 \\ i \neq j}}^{n}\left(M^{*}: x_{i}\right) \subseteq M^{*}
$$

a contradiction.

Conversely, if the vectors $x_{1}+M^{*}, x_{2}+M^{*}, \ldots, x_{n}+M^{*}$ are $\Delta$-linearly independent and there is a $j, 1<j<n$, such that $x_{j} \cap_{i=1 ; i \neq j}^{n}\left(M^{*}: x_{i}\right) \subseteq M^{*}$, then $\left(x_{j}+M^{*}\right) \cap_{i=1 ; i \neq j}^{n}\left(x_{i}+M^{*}\right)^{r}=0$ in $R^{*} / M^{*}$. Hence $\left[\Delta\left(x_{j}+M^{*}\right)\right]^{r} \supseteq$ $\left[\sum_{i=1 ; i \neq j}^{n} \Delta\left(x_{i}+M^{*}\right)\right]^{r}$ which implies that

$$
\left[\Delta\left(x_{j}+M^{*}\right)\right]^{r l} \subseteq\left[\sum_{\substack{i=1 \\ i \neq j}}^{n} \Delta\left(x_{i}+M^{*}\right)\right]^{r l}
$$

But $R^{*} / M^{*}$ is simple and so, by $\left[4\right.$, Corollary 2.2], $\left[\Delta\left(x_{j}+M^{*}\right)\right]^{r l}=\Delta\left(x_{j}+M^{*}\right)$ and $\left[\sum_{i=1 ; i \neq j}^{n} \Delta\left(x_{i}+M^{*}\right)\right]^{r l}=\sum_{i=1 ; i \neq j}^{n} \Delta\left(x_{i}+M^{*}\right)$. Thus, $\Delta\left(x_{j}+M^{*}\right) \subseteq$ $\sum_{i=1 ; i \neq j}^{n} \Delta\left(x_{i}+M^{*}\right)$, contradicting the fact that $\left\{x_{1}+M^{*}, x_{2}+M^{*}, \ldots, x_{n}+\right.$ $\left.M^{*}\right\}$ is a $\Delta$-linearly independent set of vectors.

LEMMA 4. If $R^{*}$ is quasi-projective as a right $R$-module, then every $g \in B$ gives rise to a biendomorphism $g^{*}: R^{*} / M^{*} \rightarrow R^{*} / M^{*}: x+M^{*} \rightarrow x g+M^{*}$ of $R^{*} / M^{*}$.

Proof. If $k \in \operatorname{End}\left(R^{*} / M^{*}\right)$, then $k$ lifts to $k^{\prime} \in \operatorname{End}\left(R_{R}^{*}\right)$ by the quasi-projectivity of $R^{*}$. Hence for any $x+M^{*} \in R^{*} / M^{*}$,

$$
\begin{aligned}
{\left[k\left(x+M^{*}\right)\right] g^{*} } & =\left[k^{\prime} x+M^{*}\right] g^{*} \\
& =\left(k^{\prime} x\right) g+M^{*}=k^{\prime}(x g)+M^{*}=K\left[\left(x+M^{*}\right) g^{*}\right]
\end{aligned}
$$


LEMMA 5. Let $S$ be a nonempty subset of $R^{*}-M^{*}$. If the relation $\sim$ is defined on $S$ by $x \sim y$ if there is a $k \in \operatorname{End}\left(R^{*} / M^{*}\right)$ such that $x+M^{*}=k\left(y+M^{*}\right)$, then:

(a) $\sim$ is an equivalence relation on $S$.

(b) If $\left\{x_{1}, x_{2}, \ldots, x_{n}\right\} \subseteq S$ is an $M^{*}$-independent set of elements of $R^{*}$, then the equivalence classes determined by $x_{i}$ and $x_{j}, i \neq j$, are distinct.

(c) If $R^{*}$ is quasi-projective as a right $R$-module and $x, y \in S$ are such that $x \sim y$, then $O(x, x f)=O(y, y f)$ for any $f \in B$.

Proof. Since (a) is obvious, suppose $x_{i}$ and $x_{j}, i \neq j$, determine the same equivalence class. Then $x_{i} \sim x_{j}$ and so let $k \in \operatorname{End}\left(R^{*} / M^{*}\right)$ be such that $x_{i}+M^{*}$ $=k\left(x_{j}+M^{*}\right)$. From this it follows that the set $\left\{x_{1}+M^{*}, x_{2}+M^{*}, \ldots, x_{n}+\right.$ $\left.M^{*}\right\}$ is linearly dependent. Thus, by Lemma 3 , the set $\left\{x_{1}, x_{2}, \ldots, x_{n}\right\}$ cannot be $M^{*}$-independent and so (b). To show (c), let $g \in O(x, x f)$. Then $x g-x f \in M^{*}$ and so $x(g-f)+M^{*}=0$ in $R^{*} / M^{*}$. But, by Lemma $4, g-f \in B$ gives rise to a biendomorphism $(g-f)^{*}$ of $R^{*} / M^{*}$ such that $\left(x+M^{*}\right)(g-f)^{*}=0$. Now if $k \in \operatorname{End}\left(R^{*} / M^{*}\right)$ is such that $k\left(x+M^{*}\right)=y+M^{*}$, then $k\left(x+M^{*}\right)(g-f)^{*}=$ 0 and so $\left(y+M^{*}\right)(g-f)^{*}=0$. Hence $y(g-f)+M^{*}=0$ and therefore $y g-y f$ $\in M^{*}$. Thus $g \in O(y, y f)$. Similarly any $g \in O(y, y f)$ is in $O(x, x f)$.

LEMMA 6. If $R^{*}$ is quasi-projective as a right $R$-module, then for any $f \in B$ the collection of sets $\cap_{i=1}^{n} O\left(x_{i}, x_{i} f\right)$, where $\left\{x_{1}, x_{2}, \ldots, x_{n}\right\}$ is an $M^{*}$-independent set of elements of $R^{*}$, forms a base in the $M^{*}$-topology for the neighborhood system of $f$.

Proof. To prove the lemma, it is sufficient to show that if $\cap_{i=1}^{m} O\left(y_{i}, y_{i} f\right)$ is a base element of the neighborhood system of $f$ in the $M^{*}$-topology, there is an $M^{*}$-independent set $\left\{x_{1}, x_{2}, \ldots, x_{n}\right\}$ of elements of $R^{*}$ such that $\cap_{i=1}^{n} O\left(x_{i}, x_{i} f\right)$ $\subseteq \cap_{i=1}^{m} O\left(y_{i}, y_{i} f\right)$.

First, let $y \in R^{*}$ and $f \in B$. We claim there is an $M^{*}$-independent set $\left\{x_{1}, x_{2}, \ldots, x_{n}\right\}$ of elements of $R^{*}$ such that $\cap_{i=1}^{n} O\left(x_{i}, x_{i} f\right) \subseteq O(y, y f)$. Notice that if $y \in M^{*}$, then $O(y, y f)=B$ and so if $x \in R^{*}-M^{*}$, then $O(x, x f) \subseteq$ $O(y, y f)$. Now assume $y \notin M^{*}$ and set $\Delta=\operatorname{End}\left(R^{*} / M^{*}\right)$. If $\mathscr{B}$ is a basis for the left $\Delta$-vector space $R^{*} / M^{*}$, let $y+M^{*}=\sum_{i=1}^{n} k_{i}\left(z_{i}+M^{*}\right)$ where the $z_{i}+M^{*} \in$ $\mathscr{B}$ are distinct and $0 \neq k_{i} \in \Delta$ for $i=1,2, \ldots, n$. If $x_{i}+M^{*}=k_{i}\left(z_{i}+M^{*}\right)$ for each $i$, then the set of vectors $\left\{x_{1}+M^{*}, x_{2}+M^{*}, \ldots, x_{n}+M^{*}\right\}$ is clearly $\Delta$-linearly independent. Hence, by Lemma $3,\left\{x_{1}, x_{2}, \ldots, x_{n}\right\}$ is an $M^{*}$-independent set of elements of $R^{*}$. But then $y-\sum_{i=1}^{n} x_{i} \in M^{*}$ and from this it follows that $\cap_{i=1}^{n} O\left(x_{i}, x_{i} f\right) \subseteq O(y, y f)$.

Next, suppose that $\cap_{i=1}^{m} O\left(y_{i}, y_{i} f\right)$ is a base element of the neighborhood system of $f$ where we assume, without loss of generality, that $y_{i} \notin M^{*}$ for $i=1,2, \ldots, m$. By our observations above, we see that, for each $i, 1<i<m$, there is an $M^{*}$-independent set $\left\{x_{i 1}, x_{i 2}, \ldots, x_{i n_{i}}\right\}$ of elements of $R^{*}$ such that $\bigcap_{j=1}^{n_{i}} O\left(x_{i j}, x_{i j} f\right) \subseteq O\left(y_{i}, y_{i} f\right)$ where, for each $i$ and $j, x_{i j}+M^{*}$ is a left scalar multiple of an element of $\mathscr{B}$. Hence

$$
\bigcap_{i=1}^{m} \bigcap_{j=1}^{n_{i}} O\left(x_{i j}, x_{i j} f\right) \subseteq \bigcap_{i=1}^{m} O\left(y_{i}, y_{i} f\right) .
$$


Now let $\left\{z_{1}+M^{*}, z_{2}+M^{*}, \ldots, z_{n}+M^{*}\right\}$ be the set of distinct elements of $\mathscr{B}$ such that for each $x_{i j}+M^{*}$ there are a $0 \neq k_{s} \in \Delta$ and a $z_{s}+M^{*}, 1<s<n$, such that $x_{i j}+M^{*}=k_{s}\left(z_{s}+M^{*}\right)$. Clearly such a set must exist in view of how the $x_{i j}+M^{*}$ are chosen. Now let $S=\left\{x_{i j}\right\}_{j=1}^{n_{i}} \cup_{i=1}^{m}\left\{z_{1}, z_{2}, \ldots, z_{n}\right\}$ and define the relation $\sim$ on $S$ as in Lemma 5 . If $\mathcal{E}$ denotes the equivalence classes of $S$ determined by $\sim$, then Lemma $5\left(\right.$ b) shows that $\operatorname{Card}(\mathcal{E})=n$. If $\left\{x_{1}, x_{2}, \ldots, x_{n}\right\}$ is a complete set of representatives of the equivalence classes in $\mathcal{E}$, then, by Lemma 5(c), $\cap_{i=1}^{n} O\left(x_{i}, x_{i} f\right)=\bigcap_{i=1}^{m} \bigcap_{j=1}^{n_{i}} O\left(x_{i j}, x_{i j} f\right)$. But, by Lemma 3, the set $\left\{x_{1}, x_{2}, \ldots, x_{n}\right\}$ is $M^{*}$-independent and so the proof is complete.

We are now in a position to prove the main result of this paper.

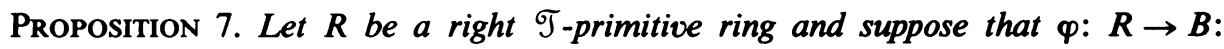
$r \rightarrow \tau_{r}$ is the canonical embedding where $B$ is endowed with the $M^{*}$-topology. If $\left\{x_{1}, x_{2}, \ldots, x_{n}\right\}$ is an $M^{*}$-independent set of elements of $R^{*}$, then for any $f \in B$, there is an $r \in R$ such that $\tau_{r} \in \cap_{i=1}^{n} O\left(x_{i}, x_{i} f\right)$. Moreover, if $R^{*}$ is quasi-projective as a right $R$-module, then $\varphi(R)$ is topologically dense in $B$.

Proof. Let $f \in B$ and suppose that $\left\{x_{1}, x_{2}, \ldots, x_{n}\right\}$ is an $M^{*}$-independent set of elements of $R^{*}$. Then, $x_{j} \cap_{i=1}^{n}\left(M^{*}: x_{i}\right) \nsubseteq M^{*}$ for $j=1,2, \ldots, n$ and so since $M^{*}$ is maximal in $R^{*}, M^{*}+\left[x_{j} \cap_{i=1 ; i \neq j}^{n}\left(M^{*}: x_{i}\right)\right]=R^{*}$ for each $j, 1<j<n$. If, for each $j, m_{j}+x_{j} t_{j}=x_{j} f$ where $m_{j} \in M^{*}$ and $t_{j} \in \cap_{i=1 ; i \neq j}^{n}\left(M^{*}: x_{i}\right)$, then $x_{j} t_{j}-x_{j} f \in M^{*}$ for $j=1,2, \ldots, n$. Now let $r=\sum_{i=1}^{n} t_{i}$, then

$$
x_{i} \tau_{r}-x_{i} f=x_{i} t_{i}-x_{i} f+\sum_{\substack{j=1 \\ j \neq i}}^{n} x_{i} t_{j} \in M^{*} \text { for } i=1,2, \ldots, n .
$$

Hence $\tau_{r} \in \cap_{i=1}^{n} O\left(x_{i}, x_{i} f\right)$. Now suppose that $R^{*}$ is quasi-projective and let $N$ be a neighborhood of $f$. By Lemma 6 , there is an $M^{*}$-independent set $\left\{x_{1}, x_{2}, \ldots, x_{n}\right\}$ of elements of $R^{*}$ such that $\cap_{i=1}^{n} O\left(x_{i}, x_{i} f\right) \subseteq N$. But, by the first part of this proposition, there is an $r \in R$ such that $\tau_{r} \in \cap_{i=1}^{n} O\left(x_{i}, x_{i} f\right)$. Hence $\tau_{r} \in \varphi(R) \cap$ $N$ and so $\varphi(R)$ is topologically dense in $B$.

As a point of interest notice that when $(\mathcal{T}, \mathcal{F})$ is the torsion theory in which every module is torsion free, $K$ is a maximal right ideal of $R$ and so $M^{*}=0$. Hence $R^{*}$ is a simple right $R$-module, $B$ is the full biendomorphism ring of $R^{*}$ and the 0 -topology on $B$ is just the finite topology on $B$. Consequently, in this setting Proposition 7 yields the usual topological version of the density theorem for right primitive rings [3, p. 31]. To see that it also yields the algebraic version [3, p. 28], let $y_{1}, y_{2}, \ldots, y_{n}$ and $x_{1}, x_{2}, \ldots, x_{n}$ be elements of $R^{*}$ and suppose that the $x_{i}$ are $\operatorname{End}\left(R^{*}\right)$-linearly independent. If $\mathscr{B}$ is a basis for $R^{*}$ such that $\left\{x_{1}, x_{2}, \ldots, x_{n}\right\} \subseteq$ $\mathscr{B}$, define $f: \mathscr{B} \rightarrow R^{*}$ to be such that $x_{i} f=y_{i}$ for $i=1,2, \ldots, n$. If $f$ is extended linearly to $R^{*}$, then the 0 -independence of the set $\left\{x_{1}, x_{2}, \ldots, x_{n}\right\}$ implies there is an $r \in R$ such that $\tau_{r} \in \cap_{i=1}^{n} O\left(x_{i}, x_{i} f\right)$. Hence $x_{i} r=x_{i} \tau_{r}=x_{i} f=y_{i}$ for $i=$ $1,2, \ldots, n$. 


\section{REFERENCES}

1. S. Dickson, A torsion theory for abelian categories, Trans. Amer. Math. Soc. 121 (1966), 223-235.

2. J. Golan, Localization of noncommutative rings, Marcel Dekker, New York, 1975.

3. N. Jacobson, Structure of rings, Amer. Math. Soc. Colloq. Publ., vol. 37, Amer. Math. Soc., Providence, R. I., rev. ed., 1964.

4. R. Johnson and E. Wong, Quasi-injective modules and irreducible rings, J. London Math. Soc. 36 (1961), 260-268.

5. J. Lambek, Torsion theories, additive semantics, and rings of quotients, Lecture Notes in Math., vol. 177, Springer-Verlag, Berlin, Heidelberg and New York, 1971.

6. Lectures on rings and modules, Blaisdell, Waltham, Mass., 1966.

7. B. Stenstrom, Rings of quotients, Springer-Verlag, Berlin, Heidelberg and New York, 1975.

Department of Mathematical Sciences, EAstern Kentucky University, Richmond, Kentucky 40475 